\title{
The Design of Smart Home for the Elderly Based on ZigBee
}

\author{
Junhan $\mathrm{He}$ \\ School of Electronics and Information, Nantong University, 226019 Nantong, China
}

\begin{abstract}
In recent years, as the pace of people's life is speeding up, more young people working far from their parents, the elderly have to live alone. With the increase of age, they tend to forget things which may create safety accidents. Therefore, a smart home system for the elderly will have good application prospects. In this paper, a smart system based on ZigBee wireless network is proposed. This system uses wireless network for transmitting information between sensor nodes and uses embedded technology to analyse the data. Once an emergency occurs, the system will send an alarm to notify the elderly. At the same time, the young people can know the situation of the house through mobile phone
\end{abstract}

\section{Introduction}

In recent years, as the pace of people's life is speeding up, more young people working far from their parents, the elderly have to live alone. As people grow older, they will be likely to forget basic things like switching off lights. And also they may forget to switch off cylinder gas causing LPG gas leakage, or close the doors resulting in financial loss. In consideration of these circumstances, the smart home for the elderly is becoming definitely important. A rather simple solution to overcome these challenges is to install huge number of sensors to monitor the environment inside the house[1].

In a smart home[2], it needs various types of sensors to achieve the information of the living environment of the elderly, such as temperature, humidity, light, noise, human infrared, smoke. The collected data is real-time displayed on the LCD of embedded system, also the embedded system analyses environmental data, and control automatically the light, air conditioning, humidification, alarm and so on[3]. The selected sensors will be introduced in the third section.

Even though many intelligent wireless sensor networks developed for specific applications have been reported[4]. ZigBee technology is employed to transmit data. The ZigBee wireless communication module can realize receiving and transmitting functions by $\mathrm{CC} 2530$. The main objective of ZigBee is for battery-powered applications where lower data rate, low cost, and longer battery life are the key requirements[5]. Therefore, the
ZigBee module based on wireless technology was used as an appropriate platform to transfer data.

The whole system uses ZigBee wireless sensor network and embedded Linux technology. The management applications are designed by the ARM processors and embedded Linux. Qt is used to design the interface of users. They can browse the data and control the whole system by touching screen which allows more convenience. Afterwards, the data is transmitted to IOS application via a Bluetooth module to display on the phone. Part 1 is mainly for background introduction; Part 2 is for system overview; Part 3 and 4 are for hardware and software implementation; Part 5 is for conclusion

\section{System overview}

As can be seen from the Figure 1, the whole system is composed of sensor nodes, coordinator, ARM and PC. These sensor nodes will be installed in the house, mainly including temperature and humidity sensors, human body infrared sensors, smoke sensors, doors and windows magnetic sensors. The data collected by each sensor is transmitted to the network coordinator using a wireless network.

The coordinator is responsible for the initiation and maintenance of the whole ZigBee network, and establishing the communication between the sensor nodes. The collected data sends through serial port to the embedded system according to a certain protocol format for a further processing. 


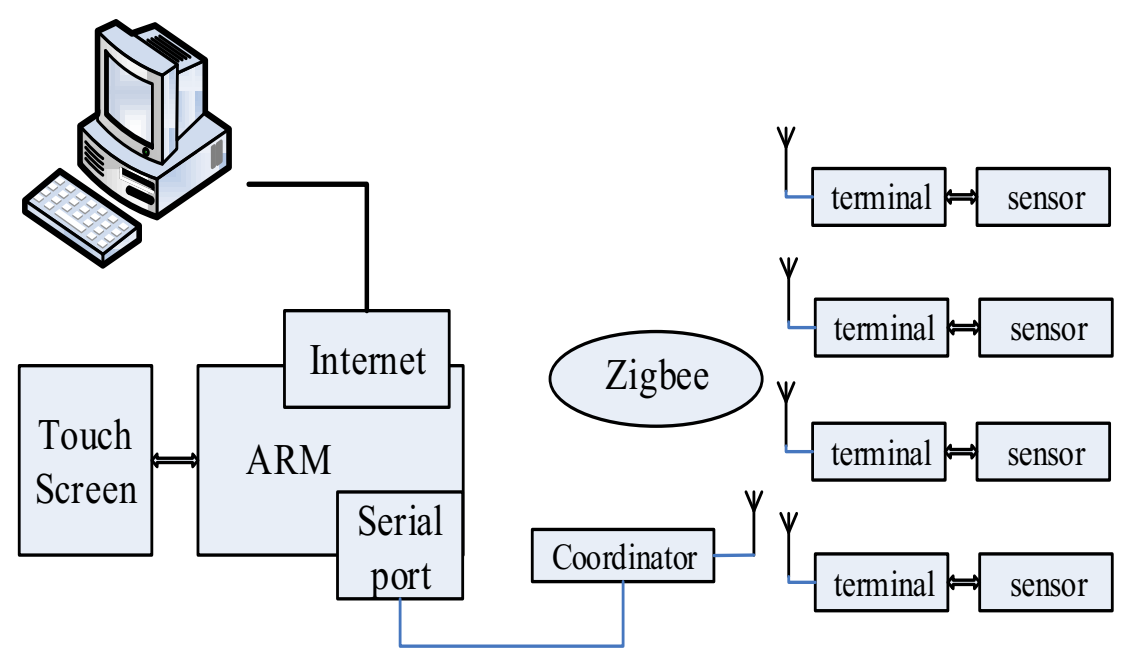

Figure 1. Block Diagram of the whole system

The embedded system collects the data from each sensor node. Each received value is processed. After that, the processed data will be in contrast with a standard threshold. When the measured data is greater than the threshold, a warning sound will be generated. At the same time, the data displays in the touch screen.

\section{Hardware implementation}

The smart home system mainly achieves the function of collecting and analysing data. It can collect and process the information of temperature, humidity, noise, smoke, the human body infrared and the door or window position. In order to achieve this goal, the next section mainly introduces the design of ZigBee communication modules and terminal node modules.

\subsection{Magnetic contact switch}

Figure 2 shows the design of the magnetic switch sensor. A voltage comparator LM393 is used in the circuit to prevent the error signal effectively caused by interference or false triggering. When the sensor is installed on the corresponding doors or windows, it can accurately detect whether a door or window is open or closed.
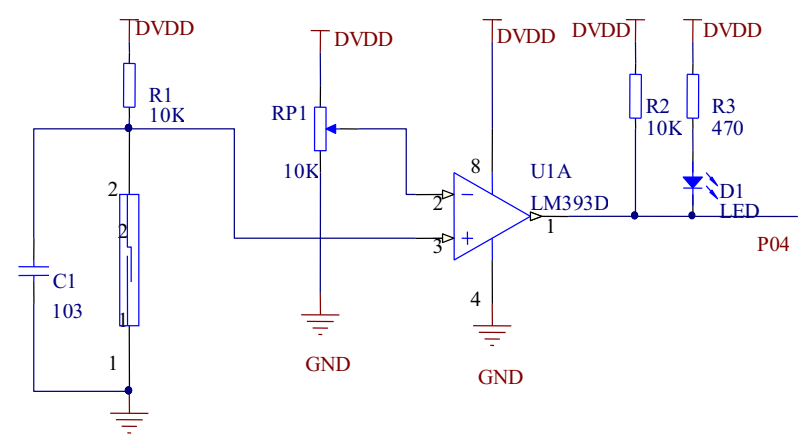

GND

Figure 2. Magnetic switch sensor

\subsection{Temperature and humidity sensor}

The temperature and humidity can reflect the comfort degree of the living environment of the elderly. SHT10 is used in the system to detect the temperature and humidity. It can work in the range of $-40 \sim 123^{\circ} \mathrm{C}$. Its error range of measuring humidity is $4.5 \% \mathrm{RH}$. Its error range of measuring temperature is $\pm 0.5^{\circ} \mathrm{C}$.

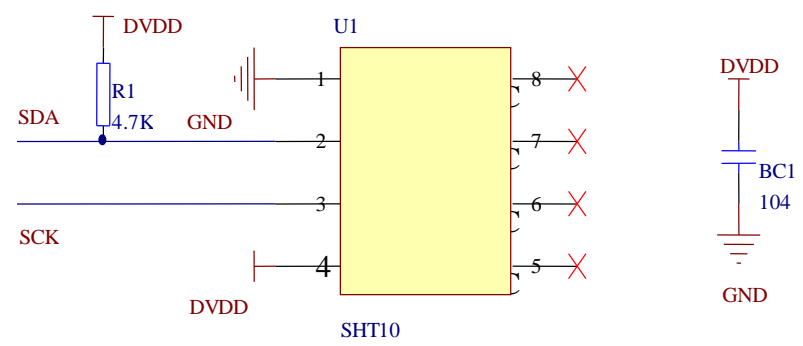

Figure 3. Circuit of temperature and humidity sensor

As shown in Figure 3, after power up, the sensor has a $11 \mathrm{~ms}$ dormant period. It needs to carry on a time delay operation. In order to decouple filter, it needs increase a $100 \mathrm{nf}$ capacitance between the power and ground.

\subsection{MQ-2 gas sensor}

When MQ-2 is in a sensitive environment, the conductivity of the sensor increases with the increase of the concentration of sensitive gas in the air, and it is suitable for the detection of liquefied gas, methane, propane, methane, alcohol, smoke and so on. As shown in the Figure 4, the adjustable resistor is used to adjust the alarm threshold. After processing, the output signal is digital. 


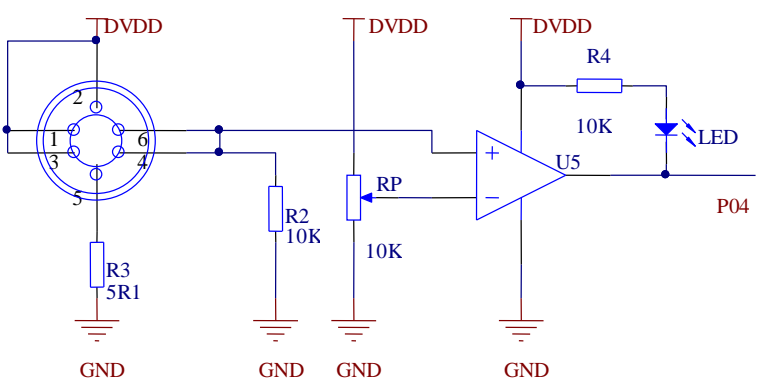

Figure 4. Circuit of gas sensor

\subsection{Noise sensor}

The noise sensor can detect environmental noise. When illegal person entering, it can detect footsteps, shattering glass, voice of conversation and then sending alarm information to inform the elderly. Due to the output of capacitor microphone is a weak signal, the system uses resistance capacitance coupling way to enlarge the signal. As shown in Figure 5, by using integrated operational amplifier LM358, the weak signal can be amplified 150 times.

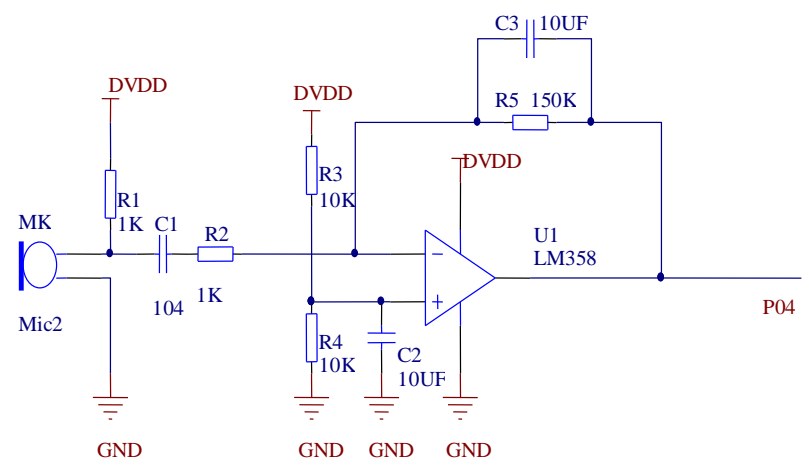

Figure 5. Circuit of noise sensor

\section{Software implementation}

\subsection{Software system of sensor nodes}

The software system of sensor nodes is mainly composed of two parts, the sensor nodes and ZigBee network communication. As shown in Figure 6, the data acquisition module is mainly responsible for sensor data collection. And the wireless communication module is mainly responsible for joining network and data sending and receiving.

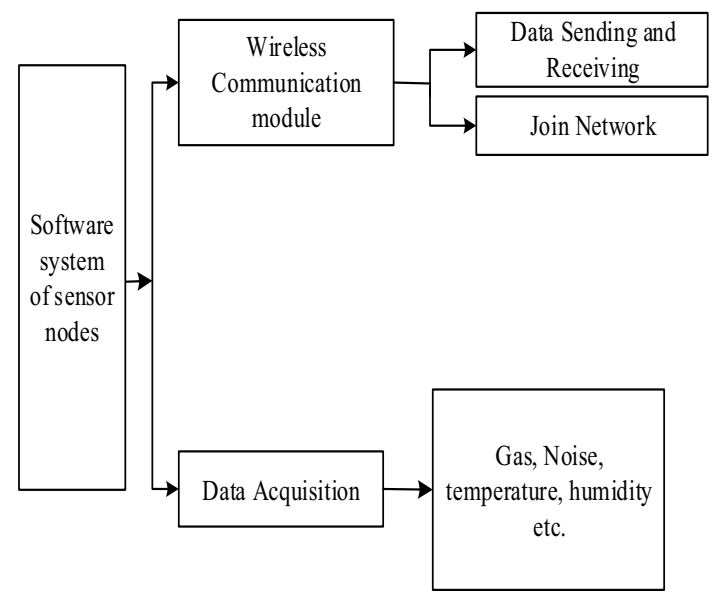

Figure 6. Software system of sensor nodes

\subsection{Program design of ZigBee terminal nodes}

The ZigBee terminal nodes mainly complete finding network, joining the network, data acquisition, sending and receiving control commands from the coordinator. The program flow chart of the ZigBee terminal node is shown in Figure 7.

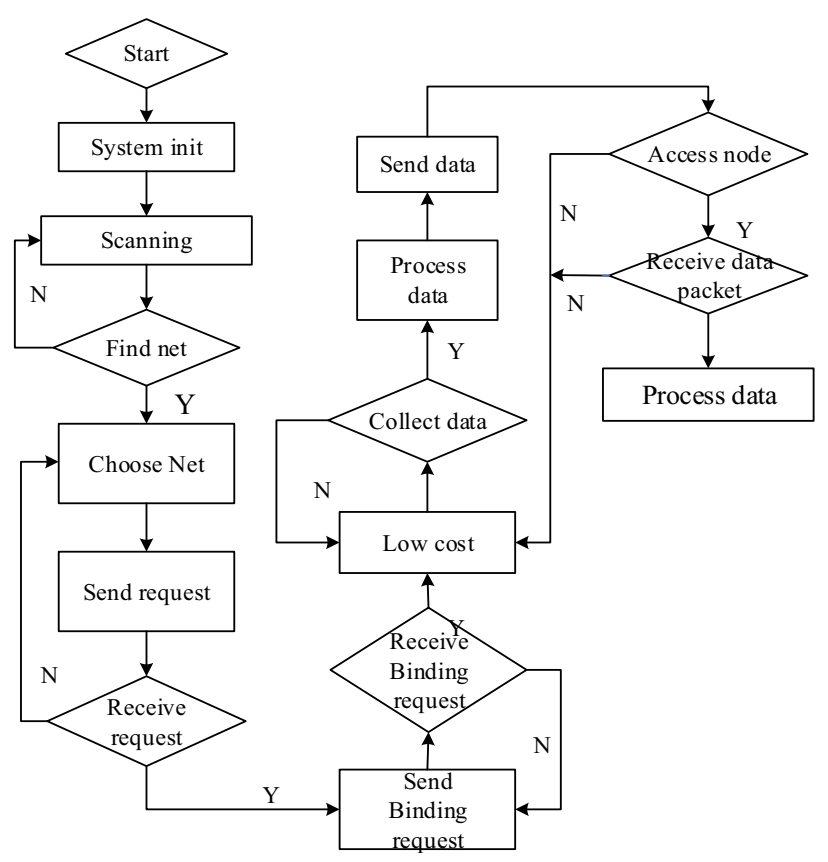

Figure 7. Flow chart of ZigBee terminal nodes 


\section{Result and conclusion}

This system is a practical application. The stability of the system and the accuracy of the data determine the quality of the system. The designs of software system and hardware system are introduced in the front sections. The whole system is shown as Figure 8.

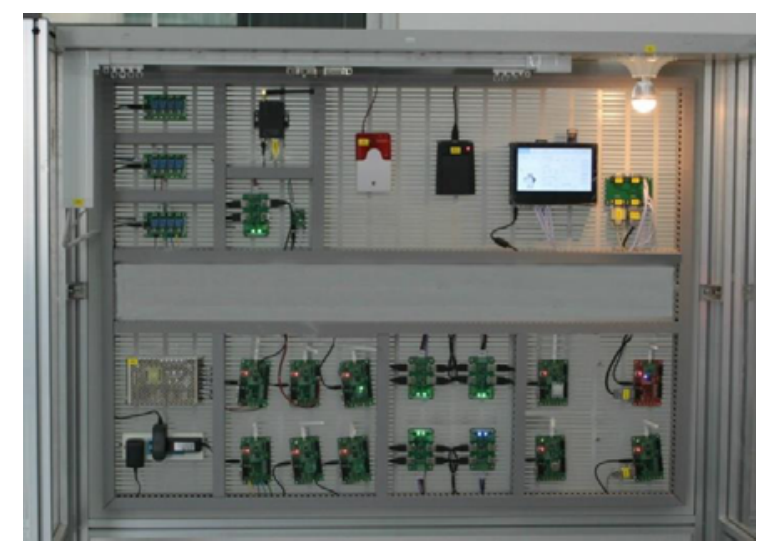

Figure 8. Structure of system

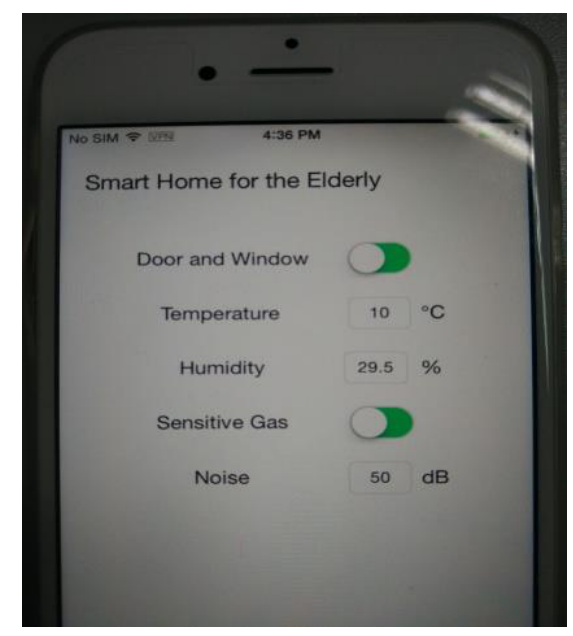

Figure 9. Data displays on the IOS device
By testing the entire system, wireless sensor nodes transmit data reliably. And the consumption of the system is low cost. The wireless transmission distance can meet the needs of the house environment. The embedded system runs stable, and interface is easy to use. The intelligent, low-cost, and low-power smart home system is realized. It has good economic efficiency and application value.

\section{References}

1. Dittmar A, Axisa F, Delhomme G, Gehin C, "New concepts and technologies in home care and ambulatory monitoring," Study Health Techno Inform, pp. 9-35(2004).

2. Ricquebourg, Vincent, David Menga, David Durand, Bruno Marhic, Laurent Delahoche, and Christophe Loge, "The smart home concept: our immediate future,” In E-Learning in Industrial Electronics, 2006 1ST IEEE International Conference on, pp. 23-28(2006).

3. N. K. Suryadevara, S. C. Mukhopadhyay, R. K. Rayudu,and Y. M. Huang, "Sensor data fusion to determine wellness of an elderly in intelligent home monitoring environment," In Proc. IEEEI2MTC Conf., Graz, Austria, pp. 947-952(2012).

4. P. Tang, T. Venables, "Smart homes and telecare for independent living," J. Telemed. Telecare 6, pp. 814(2000).

5. Rasika S. Ransing, Manita Rajput, "Smart Home for Elderly Care, based on Wireless Sensor Network," Nascent Technologies in the Engineering Field (ICNTE), 2015 International Conference on pp.1-5(2015). 